\title{
Performance of Multiple-Relay Cooperative Diversity Systems with Best Relay Selection over Rayleigh Fading Channels
}

\author{
Salama S. Ikki and Mohamed H. Ahmed \\ Faculty of Engineering and Applied Science, Memorial University, St. John's, \\ Newfoundland and Labrador, Canada A1B3X5 \\ Correspondence should be addressed to Salama S. Ikki, ikki@engr.mun.ca
}

Received 16 November 2007; Accepted 17 March 2008

Recommended by Andrea Conti

\begin{abstract}
We consider an amplify-and-forward (AF) cooperative diversity system where a source node communicates with a destination node directly and indirectly (through multiple relays). In regular multiple-relay cooperative diversity systems, all relay nodes relay the source signal using orthogonal channels (time slots, carriers, or codes) to avoid cochannel interference. Hence, for a regular cooperative diversity network with $M$ relays, we need $M+1$ channels (one for the direct link and $M$ for the $M$ indirect links). This means that the number of required channels increases linearly with the number of relays. In this paper, we investigate the performance of the best-relay selection scheme where the "best" relay only participates in the relaying. Therefore, two channels only are needed in this case (one for the direct link and the other one for the best indirect link) regardless of the number of relays $(M)$. The best relay is selected as the relay node that can achieve the highest signal-to-noise ratio (SNR) at the destination node. We show that the best-relay selection not only reduces the amount of required resources but also maintains a full diversity order (which is achievable by the regular multiple-relay cooperative diversity system but with much more amount of resources). We derive closed form expressions for tight lower bounds of the symbol error probability and outage probability. Since it is hard to find a closed-form expression for the probability density function (PDF) of SNR of the relayed signal at the destination node, we use an approximate value instead. Then, we find a closed-form expression for the moment generating function (MGF) of the total SNR at the destination. This MGF is used to derive the closed-form expressions of the performance metrics such as the average symbol error probability, the outage probability, the average SNR, the amount of fading, and the SNR moments. Furthermore, we derive the asymptotic behavior of the symbol error probability. From this asymptotic behavior, the diversity order and its dependence on the number of relays $(M)$ can be explicitly determined. Simulation results are also given to verify the analytical results.
\end{abstract}

Copyright (c) 2008 S. S. Ikki and M. H. Ahmed. This is an open access article distributed under the Creative Commons Attribution License, which permits unrestricted use, distribution, and reproduction in any medium, provided the original work is properly cited.

\section{INTRODUCTION}

Ever increasing demand for higher data rates in wireless systems has imposed serious challenges on system design and link budget planning. In many scenarios, the desired ubiquitous high rate coverage cannot be achieved by the direct transmission. Multihop relaying has emerged as an intuitive approach to this challenge. The idea is to split the distance between a source node and a destination node into several hops; the nonlinear relation between propagation loss and distance helps in reducing the end-to-end attenuation and thus in relaxing link budget. While such conventional relaying has long been known for some applications as microwave links and satellite relays, it was only until recently that this concept has received interest for wireless and mobile networks [1-5].

Cooperative diversity goes one step further, by considering the participation of several relay nodes (in addition to the source node) in delivering the signal to the destination node, to achieve diversity gain. The cooperative diversity concept is based on the following two features. First, the broadcasting nature of the wireless medium: a signal transmitted by a node propagates not only to the intended final destination, but also to other neighbor nodes. Second, viewing the individual nodes of relaying systems as distributed antennas leads to regarding cooperative diversity networks as a generalization of multiple-antenna systems. In this sense, cooperative diversity brings together 
the worlds of conventional relaying and multiple-antenna systems.

The advantages of the cooperative diversity protocols come at the expense of a reduction in the spectral efficiency since the relays must transmit on orthogonal channels in order to avoid interfering with the source node and with each other as well [6]. Hence in cooperative diversity networks with $M$ relaying nodes, $M+1$ channels are employed, which incurs a bandwidth penalty.

This problem of the inefficient use of the channel resources can be eliminated with the use of the best-relay selection scheme. In such a scheme, the "best" relay node only is selected to retransmit to the destination [7]. Hence, two channels only are required in this case (regardless of the number of relays). However, it will be shown (in Section 6) that a full diversity order (which is achievable by the regular cooperative diversity network) can still be achieved with the best-relay selection. Therefore, the efficient resource utilization by the best-relay selection scheme does not sacrifice the signal quality as will be shown later.

The best-relay selection scheme for cooperative networks has been introduced in [7], and the authors showed that this scheme has the same diversity order as the regular cooperative diversity in terms of the capacity outage. However, this important result was given using semianalytical asymptotic analysis at high SNR (without deriving a closedform expression for the capacity outage). In [8], the authors presented an asymptotic analysis (at high SNR values) only of the symbol error probability of amplify-and-forward bestrelay selection scheme, and compared it with the regular cooperative systems. The authors showed that best-relay selection scheme maintains full diversity order in terms of the symbol error probability. In [9], the authors analyzed the capacity outage probability of the best-relay selection scheme with decode-and-forward, and they showed that it outperforms distributed spacetime codes for network with more than three relaying nodes. This gain is due to the efficient use of power by the best-relay selection scheme networks. However, to the best of our knowledge, no one has derived closed-form expressions for the symbol error probability and capacity outage of the cooperative diversity network using the best-relay selection scheme at any SNR (not only high SNR values).

In this paper, we focus on nonregenerative (amplifyand-forward) dual-hop cooperative diversity network to study their end-to-end performance using the best-relay selection scheme over independent nonidentical Rayleigh fading channels. The main contribution of this paper is the derived novel closed-form expressions for the probability density function (PDF), the cumulative distribution function (CDF), and the moment generating function (MGF) of a tight lower bound value of SNR of the relayed signal at the destination. Moreover, the average symbol error probability (SEP) and the capacity outage $\left(C_{\text {out }}\right)$ are determined using closed-form expressions.

The remaining of this paper is organized as follows. Section 2 presents the system model. The analytical closedform expressions of the symbol error probability, outage capacity, and the asymptotic symbol error probability are

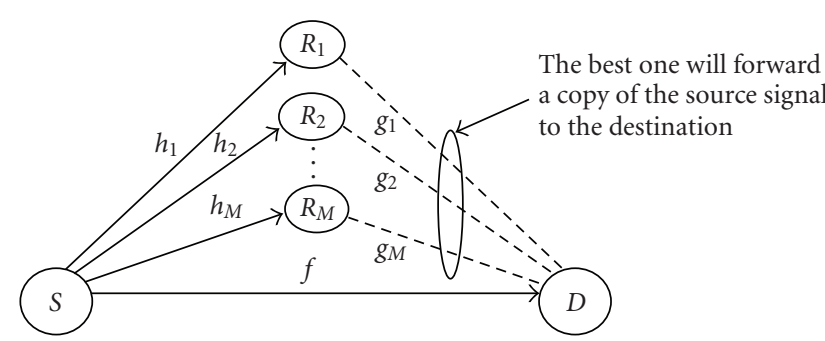

FIGURE 1: Illustration of the cooperative diversity network with the best-relay selection scheme.

derived in Sections 3, 4, and 5, respectively. Numerical results are discussed in Section 6. Finally, the conclusions are given in Section 7.

\section{SYSTEM MODEL}

As shown in Figure 1, a source node $(S)$ communicates with the destination $(D)$ through the direct link and the indirect link (through the best relay). This best-relay selection scheme allows the destination to get two copies of the source signal. The first one is from the source (direct link), while the second one is from the best relay as shown in Figure 1. The channel coefficients between the source $S$ and the $i$ th relay $R_{i}\left(h_{i}\right)$, between $R_{i}$ and $D\left(g_{i}\right)$ and between $S$ and $D(f)$ are flat Rayleigh fading coefficients. In addition, $h_{i}, g_{j}$, and $f$ are mutually-independent and nonidentical for all $i$ and $j$. We also assume here, without any loss of generality that additive white Gaussian noise (AWGN) terms of all links have zero mean and equal variance $N_{0}$.

Assuming that the relaying gain equals $\sqrt{1 /\left(E_{s} h_{i}^{2}+N_{0}\right)}$ [1] (to keep the relay power within its constraints, especially when the fading coefficient $\left(h_{i}\right)$ is low), where $E_{s}$ is the transmitted signal energy of the source, it is straightforward to show that the end-to-end SNR of the indirect link $S \rightarrow R_{i} \rightarrow D$ can be written as $[1-10]$

$$
\gamma_{S \rightarrow R_{i} \rightarrow D}=\frac{\gamma_{h_{i}} \gamma_{g_{i}}}{\gamma_{h_{i}}+\gamma_{g_{i}}+1},
$$

where $\gamma_{h_{i}}=h_{i}^{2} E_{s} / N_{0}$ is the instantaneous SNR of the source signal at $R_{i}$ and $\gamma_{g_{i}}=g_{i}^{2} E_{i} / N_{0}$ is the instantaneous SNR of the relayed signal (by $R_{i}$ ) at $D$, where $E_{i}$ is the signal transmitted energy of the relay. The best relay will be selected as the one that achieves the highest end-to-end SNR of the indirect link. Then assuming that maximum ratio combining (MRC) technique is employed at the destination node, the total SNR at the destination node can be written as

$$
\gamma_{\text {tot }}=\gamma_{f}+\max _{i}\left(\frac{\gamma_{h_{i}} \gamma_{g_{i}}}{\gamma_{h_{i}}+\gamma_{g_{i}}+1}\right),
$$

where $\gamma_{f}=f^{2} E_{s} / N_{0}$ is the instantaneous SNR between $S$ and $D$. In order to use the total SNR in the outage and error performance calculations, (1) should be expressed in a more mathematically tractable form. To achieve it, we proposed in [10] a tight upper bound for $\gamma_{S \rightarrow R_{i} \rightarrow D}$, given by

$$
\gamma_{S \rightarrow R_{i} \rightarrow D} \leq \gamma_{i}=\min \left(\gamma_{h_{i}} \gamma_{g_{i}}\right)
$$


The PDF of $\gamma_{i}$ can be expressed in terms of the average $\operatorname{SNR} \bar{\gamma}_{h_{i}}=\mathbf{E}\left(h_{i}^{2}\right) E_{s} / N_{0}$ and $\bar{\gamma}_{g_{i}}=\mathbf{E}\left(g_{i}^{2}\right) E_{s} / N_{0}($ where $\mathbf{E}(\bullet)$ is the statistical average operator) as $f_{\gamma i}(\gamma)=\left(1 / \bar{\gamma}_{i}\right) e^{-\gamma / \bar{\gamma}_{i}}$, where $\bar{\gamma}_{i}=\bar{\gamma}_{h_{i}} \bar{\gamma}_{g_{i}} /\left(\bar{\gamma}_{h_{i}}+\bar{\gamma}_{g_{i}}\right)$. Using the value of $\gamma_{i}$, we can rewrite the total SNR in (2) as

$$
\gamma_{\mathrm{tot}} \leq \gamma_{f}+\gamma_{b}
$$

where $\gamma_{b}=\max _{i}\left(\gamma_{i}\right)=\max _{i}\left(\min \left(\gamma_{h_{i}}, \gamma_{g_{i}}\right)\right)$. This approximation of the end-to-end SNR in (4) is analytically more tractable than the exact value in (2); and as a result, this facilitates the derivation of the SNR statistics (CDF, PDF, and MGF).This approximation is also adopted in many recent papers (e.g., $[7,11]$ ) and it is shown to be accurate enough, especially at medium and high SNR values as will be discussed in Section 6.

\section{ERROR PERFORMANCE ANALYSIS}

Since we assume that the MRC technique is employed at the destination, the symbol error probability (SEP) is evaluated for coherent reception only. When multichannel coherent reception is used, we can calculate SEP by averaging the multichannel conditional $\operatorname{SEP} P_{\mathrm{se}}\left(\gamma_{f}, \gamma_{b}\right)=$ $A \times \operatorname{erfc}\left(\sqrt{B\left(\gamma_{f}+\gamma_{b}\right)}\right)$, (where $\operatorname{erfc}(\cdot)$ is the complementary error function [12, Equation (8.250.4)] given by $\operatorname{erfc}(x)=$ $\left.\left(2 / \pi^{1 / 2}\right) \int_{x}^{\infty} \exp \left(-t^{2}\right) d t\right)$ over the joint random variables representing the SNR values of the direct and indirect links $\left(\gamma_{f}, \gamma_{b}\right)$. Since the random variables $\left(\gamma_{f}, \gamma_{b}\right)$ are assumed to be independent, the joint PDF $f_{\gamma_{f}, \gamma_{b}}\left(\gamma_{f}, \gamma_{b}\right)$ can be given by $f_{\gamma_{f}}\left(\gamma_{f}\right) f_{\gamma_{b}}\left(\gamma_{b}\right)$. Therefore, SEP can be determined as follows:

$$
\bar{P}_{\mathrm{se}}=\iint_{0}^{\infty} \int_{\mathrm{se}}\left(\gamma_{f}, \gamma_{b}\right) f_{\gamma_{f}}\left(\gamma_{f}\right) f_{\gamma_{b}}\left(\gamma_{b}\right) d \gamma_{f} d \gamma_{b} .
$$

Using the alternative definition of the $\operatorname{erfc}(\cdot)$ function as [13]

$$
\operatorname{erfc}(x)=\frac{2}{\pi} \int_{0}^{\pi / 2} \exp \left(-\frac{x^{2}}{\sin ^{2} \theta}\right) d \theta
$$

and by substituting (6) into (5), we obtain

$$
\begin{aligned}
\bar{P}_{\mathrm{se}}= & \int_{0}^{\infty} \int_{0}^{\infty} \frac{2}{\pi} \int_{0}^{\pi / 2} \exp \left(-\frac{B \gamma_{f}}{\sin ^{2} \theta}\right) \exp \left(-\frac{B \gamma_{b}}{\sin ^{2} \theta}\right) \\
& \times f_{\gamma_{f}}\left(\gamma_{f}\right) f_{\gamma_{b}}\left(\gamma_{b}\right) d \theta d \gamma_{f} d \gamma_{b} .
\end{aligned}
$$

Since the order of integration can be interchanged [13], we obtain

$$
\bar{P}_{\mathrm{se}}=\frac{2}{\pi} \int_{0}^{\pi / 2} M_{\gamma_{f}}\left(\frac{B}{\sin ^{2} \theta}\right) M_{\gamma_{b}}\left(\frac{B}{\sin ^{2} \theta}\right) d \theta,
$$

where $M_{\gamma_{f}}(s)=\int_{0}^{\infty} f_{\gamma_{f}}\left(\gamma_{f}\right) \exp \left(-s \gamma_{f}\right) d \gamma_{f}$ and $M_{\gamma_{b}}(s)=$ $\int_{0}^{\infty} f_{\gamma_{b}}\left(\gamma_{b}\right) \exp \left(-s \gamma_{b}\right) d \gamma_{b}$ are the MGF of $\gamma_{f}$ and $\gamma_{b}$, respectively.

In order to find $\bar{P}_{\text {se }}$, we need to find the PDF (and then the MGF) of $\gamma_{f}$ and $\gamma_{b}$. Since $f$ is Rayleigh distributed random variable, the PDF of $\gamma_{f}$ has an exponential distribution with a mean $\bar{\gamma}_{f}=\mathbf{E}\left(f^{2}\right) E_{s} / N_{0}$; hence the MGF of $\gamma_{f}$ can be easily found as

$$
M_{\gamma_{f}}(s)=\frac{1}{1+s \bar{\gamma}_{f}} .
$$

The PDF of $\gamma_{b}, f_{\gamma_{b}}(\gamma)$, can be found as follows. The CDF of $\gamma_{b}$ can be written as $F_{\gamma_{b}}(\gamma)=P\left(\gamma_{b} \leq \gamma\right)$, which can be obtained as

$$
F_{\gamma_{b}}(\gamma)=\prod_{i=1}^{M}\left(1-e^{-\gamma / \bar{\gamma}_{i}}\right) .
$$

Then the PDF can be found by taking the derivative of (10) with respect to $\gamma$, and after doing some manipulations, $f_{\gamma_{b}}(\gamma)$, can be written as

$$
\begin{aligned}
f_{\gamma_{b}}(\gamma)= & \sum_{n=1}^{M}(-1)^{n+1} \sum_{k_{1}=1}^{M-n+1} \sum_{k_{2}=k_{1}+1}^{M-n+2} \cdots \sum_{k_{n}=k_{n-1}+1}^{M} \\
& \times \prod_{j=1}^{n}\left(e^{-\gamma / \bar{\gamma}_{k_{j}}}\right) \sum_{j=1}^{n} \frac{1}{\bar{\gamma}_{k_{j}}} .
\end{aligned}
$$

By using the PDF in (11), the MGF can be written as

$$
\begin{aligned}
M_{\gamma_{b}}(s)= & \int_{0}^{\infty} e^{-s \gamma} \sum_{n=1}^{M}(-1)^{n+1} \sum_{k_{1}=1}^{M-n+1} \sum_{k_{2}=k_{1}+1}^{M-n+2} \cdots \sum_{k_{n}=k_{n-1}+1}^{M} \\
& \times \prod_{j=1}^{n}\left(e^{-\gamma / \bar{\gamma}_{k_{j}}}\right) \sum_{j=1}^{n} \frac{1}{\bar{\gamma}_{k_{j}}} d \gamma,
\end{aligned}
$$

and this integral can be evaluated in a closed form as

$$
M_{\gamma_{b}}(s)=\sum_{n=1}^{M}(-1)^{n+1} \sum_{k_{1}=1}^{M-n+1} \sum_{k_{2}=k_{1}+1}^{M-n+2} \cdots \sum_{k_{n}=k_{n-1}+1}^{M} \frac{\lambda}{s+\lambda},
$$

where $\lambda=\sum_{j=1}^{n}\left(1 / \bar{\gamma}_{k_{j}}\right)$. Substituting (13) and (9) in (8) and evaluating the integration with the help of [14, Chapter 5], $\bar{P}_{\text {se }}$ can be written in a closed form as

$$
\begin{aligned}
\bar{P}_{\text {se }}= & A \sum_{n=1}^{M}(-1)^{n+1} \sum_{k_{1}=1}^{M-n+1} \sum_{k_{2}=k_{1}+1}^{M-n+2} \cdots \sum_{k_{n}=k_{n-1}+1}^{M} \\
& \times\left(1-\frac{1 / \lambda}{1 / \lambda-\bar{\gamma}_{f}} \sqrt{\frac{B / \lambda}{1+1 / \lambda}}+\frac{\bar{\gamma}_{f}}{1 / \lambda-\bar{\gamma}_{f}} \sqrt{\frac{B \bar{\gamma}_{f}}{1+\bar{\gamma}_{f}}}\right) .
\end{aligned}
$$

\section{CAPACITY OUTAGE ANALYSIS}

The CDF of the total end-to-end SNR using the best-relay selection cooperative diversity can be found as follows [13]:

$$
F_{\gamma_{\text {tot }}}(\gamma)=\left.\mathfrak{I}^{-1}\left(M_{\gamma_{f}}(s) M_{\gamma_{b}}(s) / s\right)\right|_{s=\gamma},
$$

where $\mathfrak{I}^{-1}(\bullet)$ denotes the inverse Laplace transform. This inverse Laplace transform can be performed analytically, and the CDF of the total SNR can be expressed as (by doing 
the multiplication first and then using the partial fraction method)

$$
\begin{aligned}
F_{\gamma_{\mathrm{tot}}}(\gamma)= & \sum_{n=1}^{M}(-1)^{n+1} \sum_{k_{1}=1}^{M-n+1} \sum_{k_{2}=k_{1}+1}^{M-n+2} \cdots \sum_{k_{n}=k_{n-1}+1}^{M} \\
& \times\left(1-\frac{1 / \lambda}{1 / \lambda-\bar{\gamma}_{f}} e^{-\lambda \gamma}+\frac{\bar{\gamma}_{f}}{1 / \lambda-\bar{\gamma}_{f}} e^{-\gamma / \bar{\gamma}_{f}}\right) .
\end{aligned}
$$

The capacity outage $\left(C_{\text {out }}\right)$ is defined as the probability that the channel average mutual information $\left(I_{\text {Sel }}\right)$ falls below the required rate $R$. $C_{\text {out }}$ is a very important characterization of any cooperation protocol [1]. For the best-relay selection cooperative diversity networks, $C_{\text {out }}$ can be written as

$$
\begin{aligned}
C_{\text {out }} & =\operatorname{Pr}\left(I_{\text {sel }} \leq R\right) \\
& =\operatorname{Pr}\left(\frac{1}{2} \log _{2}\left(1+\gamma_{f}+\gamma_{b}\right) \leq R\right) \\
& =\operatorname{Pr}\left(\gamma_{f}+\gamma_{b} \leq 2^{2 R}-1\right) .
\end{aligned}
$$

Hence, $C_{\text {out }}$ is actually the CDF of $y_{\text {tot }}$ evaluated at $2^{2 R}-$ 1 ; therefore, $C_{\text {out }}=F_{\gamma_{\text {tot }}}\left(2^{2 R}-1\right)$. For a regular dualhop cooperative diversity network (without the best-relay selection scheme), it can easily be shown that $C_{\text {out }}$ is given by $C_{\text {out }}=F_{\gamma_{\text {tot }}}\left(2^{(M+1) R}-1\right)$, which is clearly greater than that of the best-relay selection scheme for $M>1$.

In order to find the other statistics of the total SNR, we have to find the PDF of $\gamma_{\text {tot }}$, which can be found directly by finding the derivative of the CDF, $F_{\gamma_{\text {tot }}}(\gamma)$, given in (16) with respect to $\gamma$ yielding

$$
\begin{aligned}
f_{\gamma_{\text {tot }}}(\gamma)= & \sum_{n=1}^{M}(-1)^{n+1} \sum_{k_{1}=1}^{M-n+1} \sum_{k_{2}=k_{1}+1}^{M-n+2} \cdots \sum_{k_{n}=k_{n-1}+1}^{M} \\
& \times\left(\frac{1}{1 / \lambda-\bar{\gamma}_{f}} e^{-\lambda \gamma}-\frac{1}{1 / \lambda-\bar{\gamma}_{f}} e^{-\gamma / \bar{\gamma}_{f}}\right) .
\end{aligned}
$$

The $l$ th moments of $\gamma_{\text {tot }}\left(\mu_{l}=\mathbf{E}\left(\gamma_{\text {tot }}^{l}\right)\right)$ can be found using (18) in a closed form as

$$
\begin{aligned}
\mu_{l}= & \Gamma(1+l) \sum_{n=1}^{M}(-1)^{n+1} \sum_{k_{1}=1}^{M-n+1} \sum_{k_{2}=k_{1}+1}^{M-n+2} \cdots \sum_{k_{n}=k_{n-1}+1}^{M} \\
& \times\left(\frac{\bar{\gamma}_{f}^{l+1}}{1 / \lambda-\bar{\gamma}_{f}}-\frac{(1 / \lambda)^{l+1}}{1 / \lambda-\bar{\gamma}_{f}}\right),
\end{aligned}
$$

where $\Gamma(\bullet)$ is the gamma function [12, Equation (8.310.1)]. By setting $l=1$ in (19), the average total SNR $\left(\bar{\gamma}_{\text {tot }}\right)$ can be obtained. Furthermore, the first two moments of $\gamma_{\text {tot }}$ can be used in order to evaluate the amount of fading $(\mathrm{AF})$ at the destination [13, Chapter 1]. The AF is defined as the ratio of the variance to the square mean of $\gamma_{\text {tot }}\left(\mathrm{AF}=\mu_{2} / \bar{\gamma}_{\text {tot }}^{2}-1\right)$.

\section{ASYMPTOTIC ANALYSIS OF THE SYMBOL ERROR PROBABILITY}

Although the expression for $\bar{P}_{\text {se }}$ in (14) enables numerical evaluation of the system performance and may not be computationally intensive, this expression does not offer insight into the effect of the different parameters (e.g., the number of relays $M$ ) that influence the system performance. In this section, we aim at expressing $\bar{P}_{\text {se }}$ in a simpler form in such a way we can see the effect of the different parameters as $\bar{\gamma}_{h_{i}}, \bar{\gamma}_{g_{i}}$ and $\bar{\gamma}_{f} \rightarrow \infty$.

The advantage of our accurate approximate solution obtained in the previous sections for the total SNR that we have a closed-form expression for the PDF. For this obtained PDF, the technique developed in [15] can be used to find asymptotic behavior of $\bar{P}_{\text {se }}$ at high SNR. If the approximate PDF of $\gamma_{f}$ and $\gamma_{b}$ can be written as $f_{\gamma_{f}}(\gamma)=a_{f} \gamma^{t_{f}}+o(\gamma)$ and $f_{y_{b}}(\gamma)=a_{b} \gamma^{t_{b}}+o(\gamma)$, respectively, where, $t_{f}$ and $t_{b}$ are positive integers, $a_{f}$ and $a_{b}$ are constants, and $o(\gamma)$ is a polynomial function of $\gamma$. For $\gamma_{f}$, the value of $a_{f}$ is $a_{f}=$ $\left(1 / \bar{\gamma}_{f}\right)$ and $t_{f}=0$ [15], for $\gamma_{b}$, the value of $a_{b}$ and $t_{b}$ can be found as follows. Using the series expansion, the CDF in (10) can be easily rewritten and approximated as

$$
\begin{aligned}
F_{\gamma_{b}}(\gamma) & =\prod_{i=1}^{M}\left(1-\left(1-\frac{\gamma}{\bar{\gamma}_{i}}+\frac{\gamma^{2}}{2 \bar{\gamma}_{i}^{2}}-\frac{\gamma^{3}}{6 \bar{\gamma}_{i}^{3}}+\cdots\right)\right) \\
& \approx \gamma \prod_{i=1}^{M} \frac{1}{\bar{\gamma}_{i}}+o(\gamma) .
\end{aligned}
$$

From (20) the values of $a_{b}$ and $t_{b}$ are as follows:

$$
a_{b}=\prod_{i=1}^{M} \frac{1}{\bar{\gamma}_{i}}, \quad t_{b}=0 .
$$
16]

Then, the approximate PDF of $\gamma_{\text {tot }}$ can be written as $[15$,

$$
f_{\gamma_{\text {tot-approx. }}}(\gamma) \approx a_{f} a_{b} \gamma+o(\gamma) .
$$

Notice that the asymptotic SEP can be given through $\bar{P}_{\mathrm{se}} \rightarrow A \int_{0}^{\infty} \operatorname{erfc} \sqrt{B \gamma} f_{\gamma_{\text {tot-approx. }}}(\gamma) d \gamma$, and after doing the integration, the asymptotic $\bar{P}_{\text {se }}$ can be written as

$$
\bar{P}_{\mathrm{se}} \longrightarrow \frac{3 A}{8 B^{2}} \frac{1}{\bar{\gamma}_{f}} \prod_{i=1}^{M} \frac{1}{\bar{\gamma}_{i}} .
$$

In order to see the effect of increasing number of branches explicitly, we assume a special case where all the channels are identical $\left(\bar{\gamma}_{1}=\bar{\gamma}_{2}=\cdots=\bar{\gamma}_{M}=\bar{\gamma}_{f}=\bar{\gamma}\right)$, then (23) can be written as $\bar{P}_{\mathrm{se}} \rightarrow 3 A /\left(8 B^{2} \bar{\gamma}^{M+1}\right)$. It can clearly be seen that the diversity order is equal to $M+1$. This means that the diversity order increases linearly with the number of relays although we use one relay only.

\section{NUMERICAL RESULTS}

In this section, we show numerical results of the analytical bit error rate (BER) for binary phase shift keying (BPSK) modulation and capacity outage $\left(C_{\text {out }}\right)$. We plot the performance curves in terms of BER and $C_{\text {out }}$ versus the SNR of the transmitted signal $\left(E_{s} / N_{0} \mathrm{~dB}\right)$, where $E_{s}$ is the transmit energy signal. We also show the results 


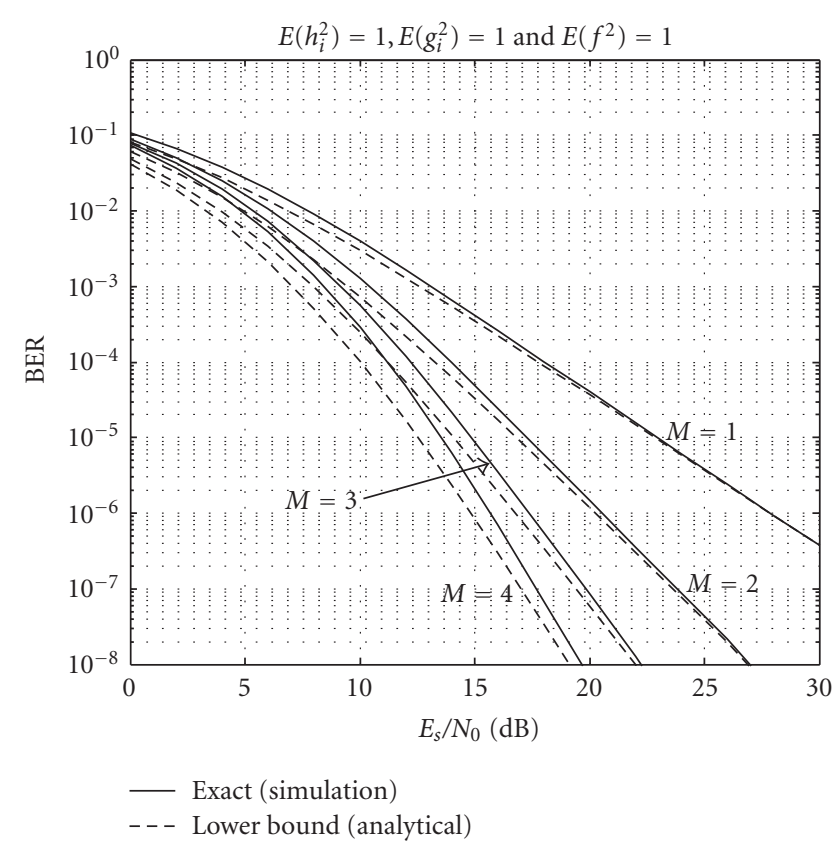

FIGURE 2: Error performance for the best-relay selection scheme over Rayleigh fading channels.

of the computer simulations of the best-relay selection scheme. We used MATLAB to build Mont-Carlo link-level simulation of the exact model shown in Figure 1 (without any approximations) to compare its results with those found from the analytical approximate model developed in the previous sections.

Figure 2 shows the BER performance of the best-relay selection scheme for different values of the number of relays $(M)$. It can be noticed form Figure 2 that the derived lower bound is tight enough, especially at medium and high SNR values. For example, the exact BER (from simulation) for $M=3$ at $E_{s} / N_{0}=15 \mathrm{~dB}$ equals $7 \times 10^{-6}$, while the analytical BER is $5 \times 10^{-6}$. This trend (the tightness of our bound) is valid at different values of $M$ as shown in Figure 2. We can also notice that the BER decreases significantly with the increase in the number of relays $(M)$ since the diversity gain and the virtual antenna gain are monotonically increasing functions of $M$.

Figure 3 shows the capacity outage $\left(C_{\text {out }}\right)$ performance for $R=1 \mathrm{bit} / \mathrm{sec} / \mathrm{Hz}$. Again, it is obvious that the derived lower bound and the simulation results are in excellent agreement. It should be noted that for Figures 2 and 3, the tightness of the derived lower bounds (for BER and $C_{\text {out }}$ ) improves as $E_{s} / N_{0}$ increases; however, both bounds (for BER and $C_{\text {out }}$ ) slightly lose their tightness at low $E_{s} / N_{0}$ values, particularly when $M$ increases. This is due to the fact that the accuracy of total SNR approximation (in (4)) improves as $E_{s} / N_{0}$ increases. From Figures 2 and 3 , it is evident that the diversity order is equal to $M+1$, which verifies the asymptotic analysis.

Figures 4 and 5 compare the performance of the bestrelay selection scheme and the regular cooperative diversity in terms of the BER and $C_{\text {out }}$ for different values of $M$. To make

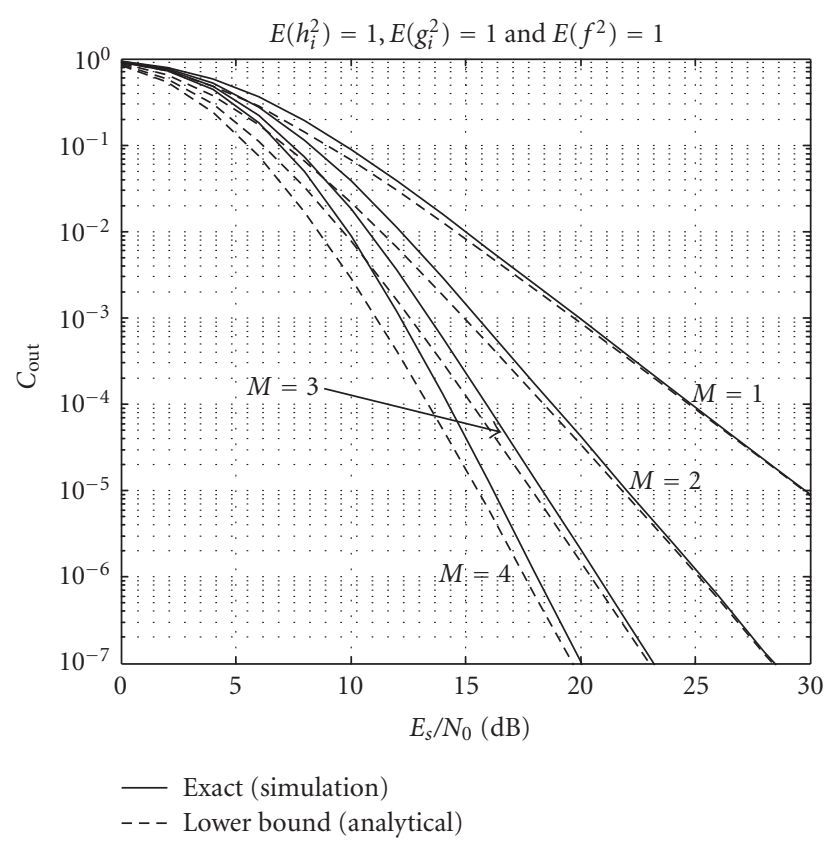

FIGURE 3: Outage performance for path the best-relay selection scheme over Rayleigh fading channels.

the comparisons fair, the transmitted power of the $M+1$ transmitting nodes (the source node plus the $M$ relays) in the regular cooperative system is set to $E_{s}=E_{i}=1 /(M+$ $1)$. For the best-relay selection scheme, we have only two node (the source and the best relay), so $E_{s}=E_{1}=1 / 2$. From Figure 4, we can see an interesting result that the bestrelay selection cooperative diversity scheme outperforms the regular cooperative diversity in terms of the BER. Also, we can see that as $M$ increases this improvement also increases. This behavior is due to the efficient use of power by the bestrelay selection scheme.

Figure 5 depicts the outage capacity for $R=1 \mathrm{bit} / \mathrm{sec} / \mathrm{Hz}$. Figure 5 shows the dramatic improvement of the best-relay selection cooperative diversity over the regular one in terms of capacity outage. In this figure, as $M$ increases, the capacity outage of the regular cooperative diversity does not necessarily improve. Actually, at low and medium SNR values the capacity outage increases. This is due to the fact that with regular cooperative diversity networks, when the number of relays increases, more channels are needed for relaying; hence it becomes more difficult to achieve the required rate $(R)$. This behavior is completely avoided in the best-relay selection scheme because we need only two orthogonal channels for transmissions regardless of the number of relays. Hence increasing the number of relays in the best-relay selection scheme always improves the capacity outage without any additional channel resources. This improvement does not depend on the value of $E_{s} / N_{0}$, unlike regular cooperative networks, where the value of $E_{s} / N_{0}$ determines whether increasing the number of relays will decrease the capacity outage or not. For instance, increasing the number of relays $(M)$, from 1 to 2 , will reduce $C_{\text {out }}$ regardless of the value of $E_{s} / N_{0}$, if the best-relay selection scheme is used. However, if 


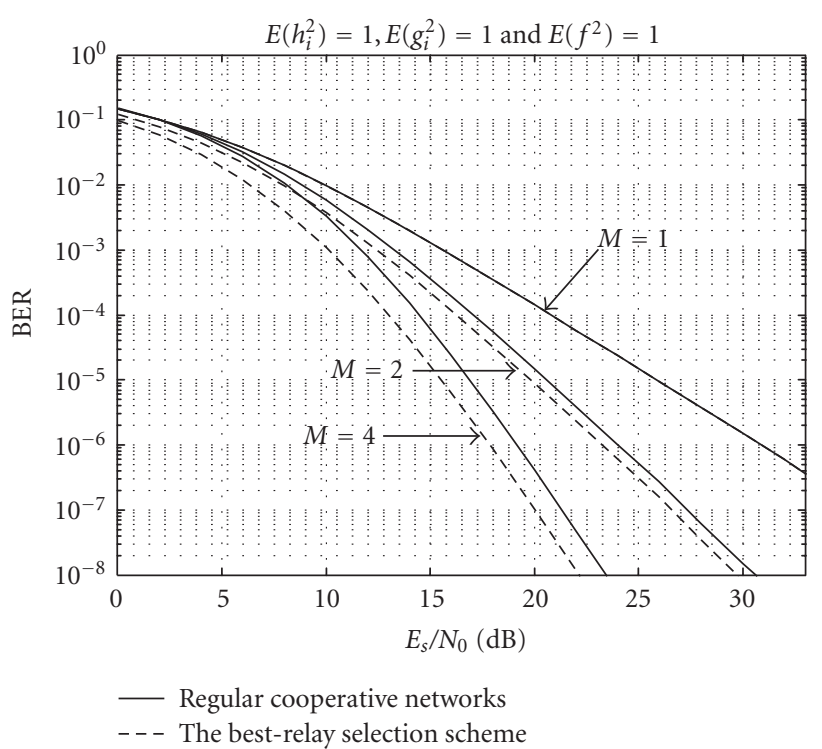

FIgURE 4: Comparison between the regular cooperative diversity and the best-relay selection scheme over Rayleigh fading channels. (Note that for $M=1$, the regular cooperative diversity and bestrelay selection scheme are the same.)

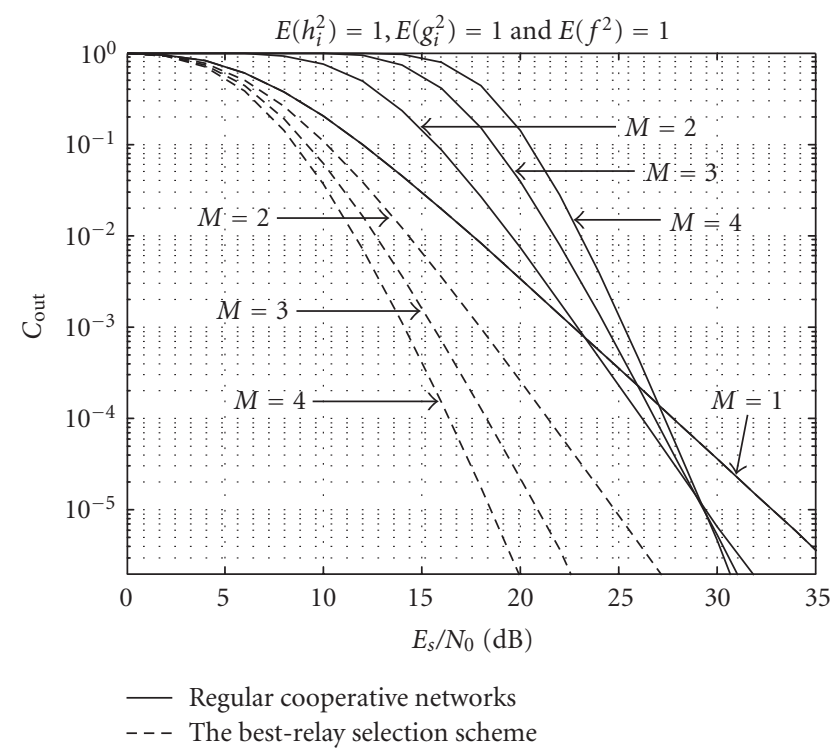

FIgURE 5: Comparison between the regular cooperative diversity and the best-relay selection scheme over Rayleigh fading channels. (Note that for $M=1$, the regular cooperative diversity and bestrelay selection scheme are the same.)

regular cooperative diversity is employed, increasing $M$ from 1 to 2 reduces $C_{\text {out }}$ only if $E_{s} / N_{0}>23 \mathrm{~dB}$.

\section{CONCLUSION}

We have analyzed the performance of the best-relay selection scheme for cooperative diversity networks operating over independent but not necessarily identically distributed
Rayleigh fading channels. Novel closed-form expressions for the average SNR, amount of fading, symbol error probability, and capacity outage were derived for any range of SNR (not only high SNR values). Computer simulation results verified the accuracy and the correctness of the derived expressions. We can conclude that best-relay selection scheme offers full diversity order.

It should be emphasized that the best-relay selection scheme has a strong advantage in saving the channel resources compared to regular cooperative diversity networks. Since the former the total capacity is reduced by $50 \%$ only while the latter reduced the channel by $1 / M$. This means that in path-selection system increasing $M$ will increase the diversity order without decreasing the channel capacity.

The only disadvantage of this system is the need for a mechanism to find the best relay; however, the implementation of best-relay selection scheme can be achieved with minimal signaling overhead and minor additional complexity as shown in [7]. As a future work, our analysis will be extended to the decode-and-forward relaying technique.

\section{REFERENCES}

[1] M. O. Hasna and M.-S. Alouini, "End-to-end performance of transmission systems with relays over Rayleigh-fading channels," IEEE Transactions on Wireless Communications, vol. 2, no. 6, pp. 1126-1131, 2003.

[2] M. O. Hasna and M.-S. Alouini, "Harmonic mean and end-toend performance of transmission systems with relays," IEEE Transactions on Communications, vol. 52, no. 1, pp. 130-135, 2004.

[3] M. O. Hasna and M.-S. Alouini, "Outage probability of multihop transmission over Nakagami fading channels," IEEE Communications Letters, vol. 7, no. 5, pp. 216-218, 2003.

[4] G. K. Karagiannidis, "Performance bounds of multihop wireless communications with blind relays over generalized fading channels," IEEE Transactions on Wireless Communications, vol. 5, no. 2, pp. 498-503, 2006.

[5] G. K. Karagiannidis, T. A. Tsiftsis, and R. K. Mallik, "Bounds for multihop relayed communications in Nakagami- $m$ fading," IEEE Transactions on Communications, vol. 54, no. 1, pp. 18-22, 2006.

[6] J. N. Laneman, D. N. C. Tse, and G. W. Wornell, "Cooperative diversity in wireless networks: efficient protocols and outage behavior," IEEE Transactions on Information Theory, vol. 50, no. 12, pp. 3062-3080, 2004.

[7] A. Bletsas, A. Khisti, D. P. Reed, and A. Lippman, "A simple cooperative diversity method based on network path selection," IEEE Journal on Selected Areas in Communications, vol. 24, no. 3, pp. 659-672, 2006.

[8] Y. Zhao, R. Adve, and T. J. Lim, "Symbol error rate of selection amplify-and-forward relay systems," IEEE Communications Letters, vol. 10, no. 11, pp. 757-759, 2006.

[9] E. Beres and R. S. Adve, "Selection Cooperation in MultiSource Cooperative Networks," IEEE Transactions on Wireless Communications, vol. 7, no. 1, pp. 118-127, 2008.

[10] S. S. Ikki and M. H. Ahmed, "Performance analysis of cooperative diversity wireless networks over Nakagami- $m$ fading channel," IEEE Communications Letters, vol. 11, no. 4, pp. 334-336, 2007. 
[11] T. Wang, A. Cano, G. B. Giannakis, and J. N. Laneman, "High-performance cooperative demodulation with decodeand-forward relays," IEEE Transactions on Communications, vol. 55, no. 7, pp. 1427-1438, 2007.

[12] I. S. Gradshteyn and I. M. Ryzhik, Table of Integrals, Series and Products, Academic Press, San Diego, Calif, USA, 5th edition, 1994.

[13] M. K. Simon and M.-S. Alouini, Digital Communication over Fading Channels, John Wiley \& Sons, New York, NY, USA, 2000.

[14] A. Papoulis, Probability, Random Variables, and Stochastic Processes, McGraw-Hill, New York, NY, USA, 1991.

[15] Z. Wang and G. B. Giannakis, "A simple and general parameterization quantifying performance in fading channels," IEEE Transactions on Communications, vol. 51, no. 8, pp. 13891398, 2003.

[16] J. Proakis, Digital Communications, McGraw-Hill, New York, NY, USA, 4th edition, 2001. 